\title{
Geometric Flow and Bandelet Coefficient Combining Method is Applied to Image Fusion
}

\author{
Mantian Zhong \\ Department of Education, Luoding Polytechnic, Luoding , 527200,China \\ email: 409490472@qq.com
}

\begin{abstract}
Keywords: The Combination of Geometric Flow and Bandelet Coefficients, Image Fusion, Bandelet Transform
\end{abstract}

\begin{abstract}
In order to explore and test the geometric flow and Bandelet coefficient combining method is applied to image fusion effect, in this thesis, on the basis of the Bandelet transform theory, with the help of Beamlab200 toolbox and Matlab platform, using construct geometric flow and Bandelet coefficients combining method and computer simulation method, from the simulation experiment it is concluded that the method in the aspect of image fusion at run time, convergence speed, standard deviation, signal-to-noise ratio and gradient more superior than Beamlab200 toolbox methods.
\end{abstract}

\section{Introduction}

Image fusion is to use a certain method of two image to form a new image fusion, image fusion can be used in the processing of remote sensing image, the process can be applied to western medicine image, also can be used in the visual image of processing network, and gradually developed a fast developing area of research. When introducing Bandelet transform image fusion, it can gather some useful information reflect the characteristics of the source image, and these information can offer a lot of great help for image fusion experiments. To do this here introduce smaller value rules, geometric flow and Bandelet coefficients combining methods for image fusion and obtained the very good fusion effect.[1].

\section{Geometric Regular Images and Geometric Flow[2][3]}

Assume that the support area $[0,1]^{2}$ of the image $f(x, y),[0,1]^{2}$ is only a continuous uninterrupted edge and with $\Gamma$ said, the square area continuous edge $\Gamma$ is with limited length of geometry. In order to convenient said, here it is assumed that the $\Gamma$ is in the $x$ projection interval on the shaft $[a, b]$, and the $\Gamma$ has the following form

$$
\Gamma=\{(x, \gamma(x)) \mid a \leq x \leq b\}
$$

$$
\text { Making } \Omega=[0,1]^{2}-\Gamma, \mathrm{C}^{\alpha}(\Omega) \text { express } \alpha \text { rank Hölder space. If } f(x, y) \in C^{\alpha}(\Omega) \text {, }
$$
$\gamma(x) \in C^{\alpha}[a, b]$, so the image $f(x, y)$ is a $\alpha$ rank geometrically regular image. The removal of $f(x, y)$ is a piece wise smooth edge line, but in other areas is smooth. Taking into account any edges in natural images have a certain width, then the $f(x, y)$ can be corrected for

$$
f=\tilde{f} * h
$$

Among them $\tilde{f} \in C^{\alpha}[a, b], \Omega=[0,1]^{2}-\Gamma \cdot h(x)$ is a compact support fuzzy kernel of an unknown. 
Given a regular image, it change is carried out along the edge parallel to the line direction ; but the change of image would be more violent is perpendicular to the direction of the edge. Usually using the geometric flow represent parallel to the direction of the edge line. In fact, the geometric flow is a definition in the image $f$ the support region of the vector field $v\left(x_{1}, x_{2}\right)$, express $f$ at every point $\left(x_{1}, x_{2}\right)$ in regular change of direction. The basic starting point of Bandelet is to make full use of the image transform along the regular geometry flow to represent the best sparse image.

\section{The Optimal Geometric Flow}

Assume $\mathrm{S}$ is a square area, along the geometrical flow direction $\mathrm{d}$ is Bandelet coefficients in one-dimensional signal of rearrangement $S$, if select the appropriate of the direction $\mathrm{d}$, then the one-dimensional hyper wavelet transform can further compression of the original signal. However, if select $\mathrm{S}$ and $\mathrm{d}$ is incorrect, the one dimensional hyper wavelet zero coefficient, number of non quantized won't have a significantly reduced. Considering the comprehensive code of two direction: bit rate and distortion, the regional of $S$ is the optimal geometric flow in the direction, should take the minimum Lagrangian function [4][5].

$$
\left(f_{\theta}, \mathrm{R}\right)=\left\|f_{\theta}-\tilde{f}_{\theta}\right\|^{2}+\lambda * T^{2}\left(R_{g}+R_{b}\right)
$$

In the formula (3) the $\tilde{f}_{\theta}$ is represented by quantized Bandelet coefficient of one-dimensional signal reconstruction, $\mathrm{T}$ is seem as the quantization threshold, $\mathrm{R}_{g}$ express bit encoding geometric flow required number, $\mathrm{R}_{b}$ express coding of the quantized Bandelet coefficient of the number of bits required. $\lambda$ is Lagrange multiplier in accordance with the Pennec results, and in the experiment $\lambda=5 / 28$.

To the small squares of $L \times L$, it can put a fillet $[0, \pi)$ discrete $L^{2}-1$ number equal angles, that is $\theta$ possible values for

$$
\theta=\frac{k \pi}{L^{2}-1}, k=0,1,2, \cdots, L^{2}-2
$$

But for the non geometric flow situation, marking $\theta=\operatorname{Inf}$, tags to show here is not implemented Bandele.

Bandelet need to adopt uniform quantization coefficient, for this, it is assumed that $x$ express Bandelet coefficient, $T$ as the quantization threshold, so the quantitative value $Q(x)$ can express as

$$
\mathrm{Q}(\mathrm{x})=\left\{\begin{array}{l}
0,|\mathrm{x}| \leq T \\
\operatorname{sgn}(x)\left(q+\frac{1}{2}\right) T, q T \leq|x| \leq(q+1) T
\end{array}\right.
$$

\section{Geometric Flow and Coefficient of Bandelet Combination Rule}

Here are the Bandelet transform image fusion procedures as follows:

(1)The two original images is the geometric registration;

(2)In the region $\Omega_{i}$, calculation of the sample image resampling;

(3)Calculation the geometry flow $\mathrm{G}_{j}(i),(j=1,2, \cdots, N)$ of each region and its corresponding Bandelet coefficient. $N$ represent the total number of original image, $\mathrm{G}_{j}(x, y, i)$ is pixel $(x, y)$ of the $j$ th original image Bandelet coefficient 
(4)Fusion rules are as follows

(1)For the geometric flow, minimum fusion rule

$\mathrm{G}_{\mathrm{F}}(i)=\left\{\begin{array}{l}\mathrm{G}_{1}(i), \mathrm{G}_{1}(i) \leq \mathrm{G}_{2}(i) \\ \mathrm{G}_{2}(i), \mathrm{G}_{1}(i)>\mathrm{G}_{2}(i)\end{array}\right.$

(2)For the Bandelet coefficient use the minimum absolute value fusion rule

$\mathrm{G}_{F}(x, y, i)=\left\{\begin{array}{l}\mathrm{G}_{1}(x, y, i),\left|\mathrm{G}_{1}(x, y, i)\right| \leq\left|\mathrm{G}_{2}(x, y, i)\right| \\ \mathrm{G}_{2}(x, y, i),\left|\mathrm{G}_{1}(x, y, i)\right|>\left|\mathrm{G}_{2}(x, y, i)\right|\end{array}\right.$

In the formula (6) and (7), $\mathrm{G}_{\mathrm{F}}(i)$ express the fused image $\Omega$ regional geometric flow, $\mathrm{G}_{F}(x, y, i)$ express the fused image at pixel point $(x, y)$ 's Bandelet coefficient.

(5)By using the geometric flow $\mathrm{G}_{\mathrm{F}}(i)$ and Bandelet coefficient $\mathrm{G}_{F}(x, y, i)$ carry out Bandelet reconstruction transform of image fusion.

\section{Comparing the Simulation Experiment of Time Efficiency}

Table 1 is the comparison of using Beamlab200 toolbox method, Geometric flow and Bandelet coefficient combining method with running Matlab7.01 platform running time.

Table 1 Comparison of using Beamlab200 toolbox method, Geometric flow and Bandelet coefficient combining method with running Matlab7.01 platform running time.

\begin{tabular}{ccc}
\hline $\begin{array}{c}\text { Fusion } \\
\text { method }\end{array}$ & $\begin{array}{c}\text { Beamlab200 } \\
\text { toolbox method }\end{array}$ & $\begin{array}{c}\text { Geometric flow and Bandelet } \\
\text { coefficient method combine }\end{array}$ \\
\hline running time & 593seconds & 386 seconds \\
\hline
\end{tabular}

\section{Comparing the Simulation Experiment of Image Effect}

Fig.1 is compared with Beamlab200 toolbox method and Bandelet, geometric flow coefficient combining method with operation platform of Matlab7.01 convergence speed.

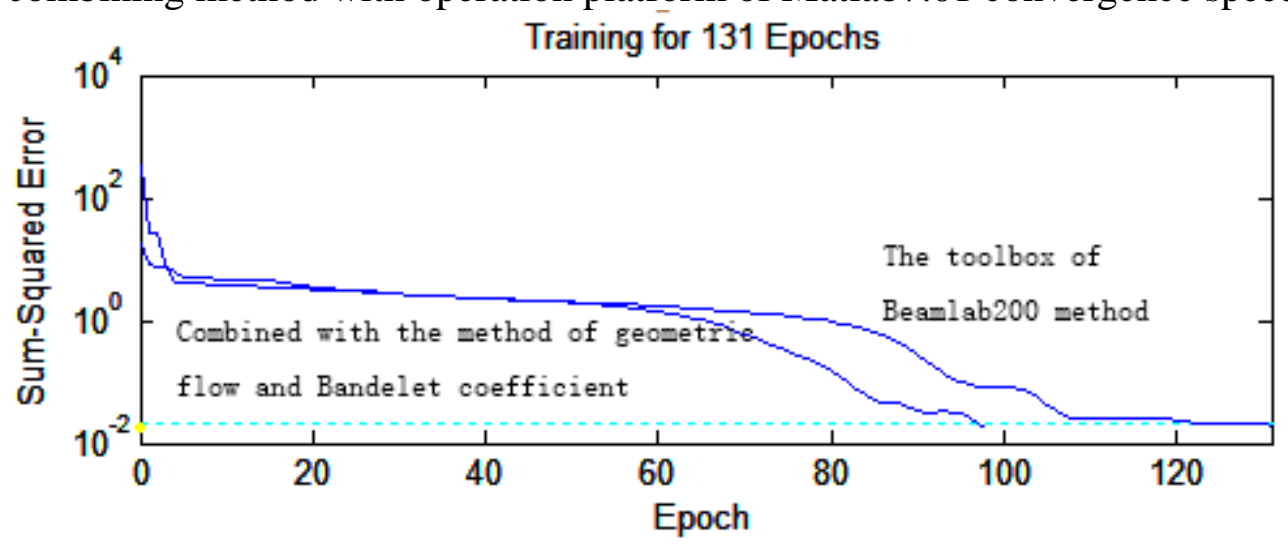

Fig.1. two method convergence speed

\section{Parameter Comparison}

Table 2 are two kinds of methods to use the Beamlab200 toolbox method, geometric flow and Bandelet coefficient combining methodwithMatlab7.01 platform are compared in terms of the mean square error, signal to noise ratio, the number of iterations, the average gradient and entropy etc. five aspects. 
Table 2 comparison of Beamlab200 toolbox and geometric flow and Bandelet coefficient combining method with parameters

\begin{tabular}{|c|c|c|c|c|c|}
\hline $\begin{array}{l}\text { Image } \\
\text { fusion method name }\end{array}$ & $\begin{array}{l}\text { Standard } \\
\text { deviation }\end{array}$ & $\begin{array}{l}\text { Signal to } \\
\text { noise } \\
\text { ratio }(\mathrm{db})\end{array}$ & $\begin{array}{l}\text { The } \\
\text { number of } \\
\text { iterations }\end{array}$ & $\begin{array}{l}\text { The } \\
\text { average } \\
\text { gradient }\end{array}$ & Entropy \\
\hline $\begin{array}{l}\text { The toolbox of } \\
\text { Beamlab200 method }\end{array}$ & 71.4153 & 38.6529 & 131 & 19.6584 & 7.7583 \\
\hline $\begin{array}{l}\text { combining with } \\
\text { the method of } \\
\text { geometric flow and } \\
\text { Bandelet coefficient }\end{array}$ & 69.2548 & 40.0058 & 96 & 18.5389 & 7.7695 \\
\hline
\end{tabular}

\section{Conclusion}

According to table data comparison in table 1 can get to know, two methods of difference in the running time is not too large, the Geometric flow and Bandelet coefficients combining method more faster about 200 seconds than Beamlab200 method; according to the figure 1 shows, the geometric flow and Bandelet coefficient combining method with fast in convergence speed; according to table 2 data can get to know, comparing a toolbox of Beamlab200 method, the geometric flow and Bandelet coefficient combining method in the standard deviation of reduced 2,increase signal-to-noise ratio 1.4,training steps to reduce nearly 40 step, the average gradient decrease 1,entropy is almost. All in all, geometric flow and Bandelet coefficients combining method in the aspect of image fusion has a better fusion result.

\section{References}

[1] Erwan Le Pannec, Stephane Mallat. Bandelet Image Approximation and Compression. SIAM Journal of Multiscale Modeling and Simulation,sol.4.no.3,pages992-1039,2005.

[2] Jingwen Yan, Xiaobo Qu. The super wavelet analysis and application [M]. National Defence Industry Press, 2008

[3] Bowles, Guosheng Rui, Zhengxing Cheng, Wen Wang . Wavelet and wavelet transform introduction [M]. Publishing House of electronics industry,2013

[4] Yankui Sun. Wavelet transform and image, graphics processing technology [M]. Tsinghua University press, 2012

[5] T.K. Moon and W.C. Stirling, Mathematical Methods and Algorithms for Signal Processing. New Jensey,NJ:Prentice,2000.

[6] Defeng Zhang. MATLAB hyper wavelet analysis [M]. Mechanical Industry Press, 2012 (2) 